\title{
Postsynaptic structure formation of human iPS cell-derived neurons takes longer than presynaptic formation during neural differentiation in vitro
}

\author{
Kazuyuki Togo 1,2, Hayato Fukusumi ${ }^{2}$, Tomoko Shofuda ${ }^{2}$, Hiroshi Ohnishi ${ }^{3}$, Hiroyuki Yamazaki ${ }^{4,5}$, \\ Mariko Kato Hayashi, ${ }^{6,7}$, Nana Kawasaki ${ }^{8}$, Nobuyuki Takei ${ }^{9}$, Takanobu Nakazawa ${ }^{10,11}$, Yumiko Saito ${ }^{12}$, \\ Kousuke Baba ${ }^{1}$, Hitoshi Hashimoto ${ }^{10,13,14,15,16}$, Yuko Sekino ${ }^{17}$, Tomoaki Shirao ${ }^{4}$, Hideki Mochizuki ${ }^{1}$ and \\ Yonehiro Kanemura ${ }^{18,19^{*}}$ (1)
}

\begin{abstract}
The generation of mature synaptic structures using neurons differentiated from human-induced pluripotent stem cells (hiPSC-neurons) is expected to be applied to physiological studies of synapses in human cells and to pathological studies of diseases that cause abnormal synaptic function. Although it has been reported that synapses themselves change from an immature to a mature state as neurons mature, there are few reports that clearly show when and how human stem cell-derived neurons change to mature synaptic structures. This study was designed to elucidate the synapse formation process of hiPSC-neurons. We propagated hiPSC-derived neural progenitor cells (hiPSC-NPCs) that expressed localized markers of the ventral hindbrain as neurospheres by dual SMAD inhibition and then differentiated them into hiPSC-neurons in vitro. After 49 days of in vitro differentiation, hiPSC-neurons significantly expressed pre- and postsynaptic markers at both the transcript and protein levels. However, the expression of postsynaptic markers was lower than in normal human or normal rat brain tissues, and immunostaining analysis showed that it was relatively modest and was lower than that of presynaptic markers and that its localization in synaptic structures was insufficient. Neurophysiological analysis using a microelectrode array also revealed that no synaptic activity was generated on hiPSC-neurons at 49 days of differentiation. Analysis of subtype markers by immunostaining revealed that most hiPSC-neurons expressed vesicular glutamate transporter 2 (VGLUT2). The presence or absence of NGF, which is required for the survival of cholinergic neurons, had no effect on their cell fractionation. These results suggest that during the synaptogenesis of hiPSC-neurons, the formation of presynaptic structures is not the only requirement for the formation of postsynaptic structures and that the mRNA expression of postsynaptic markers does not correlate with the formation of their mature structures. Technically, we also confirmed a certain level of robustness and reproducibility of our neuronal differentiation method in a multicenter setting, which will be helpful for future
\end{abstract}

\footnotetext{
*Correspondence: kanemura.yonehiro.hk@mail.hosp.go.jp

${ }^{18}$ Division of Regenerative Medicine, Department of Biomedical Research and Innovation, Institute for Clinical Research, National Hospital Organization Osaka National Hospital, 2-1-14 Hoenzaka, Chuo-ku, Osaka, Osaka 540-0006, Japan
}

Full list of author information is available at the end of the article permits use, sharing, adaptation, distribution and reproduction in any medium or format, as long as you give appropriate credit to the original author(s) and the source, provide a link to the Creative Commons licence, and indicate if changes were made. The images or other third party material in this article are included in the article's Creative Commons licence, unless indicated otherwise in a credit line to the material. If material is not included in the article's Creative Commons licence and your intended use is not permitted by statutory regulation or exceeds the permitted use, you will need to obtain permission directly from the copyright holder. To view a copy of this licence, visit http://creativecommons.org/licenses/by/4.0/. The Creative Commons Public Domain Dedication waiver (http://creativeco mmons.org/publicdomain/zero/1.0/) applies to the data made available in this article, unless otherwise stated in a credit line to the data. 
research. Synapse formation with mature postsynaptic structures will remain an interesting issue for stem cell-derived neurons, and the present method can be used to obtain early and stable quality neuronal cultures from hiPSC-NPCs.

Keywords: Human-induced pluripotent stem cell, Neural progenitor cell, Vesicular glutamate transporter 2 (VGLUT2), Drebrin, PSD-95

\section{Background}

Neural progenitor cells (NPCs) can be differentiated from human-induced pluripotent stem cells (hiPSCs) using various methods [1]; they are self-renewing and can differentiate into various types of neurons. Mature neurons induced from hiPSC-NPCs are useful for the physiological, pathological, and pharmacological characterization of human neurons because their accessibility is not limited and because they are free from ethical issues regarding the preparation of neuronal cultures from the human brain [2-6]. With this background, in vitro application of stem cell-derived neurons has been expected, and methods to induce differentiation into various types of neurons have been developed. Stem cell-derived neurons are also expected to be applied to synapses, which transmit information between neurons. There are many neurological diseases that are believed to be related to synaptic dysfunction, such as Alzheimer's disease, Huntington's disease, and Parkinson's disease [7].

In neural tissue, a substantial amount of excitatory synaptic transmission is mediated by glutamate. Vesicular glutamate transporters carry glutamate to synaptic vesicles, and three isoforms have been identified in mammals, including humans: vesicular glutamate transporter 1 (VGLUT1), vesicular glutamate transporter 2 (VGLUT2), and vesicular glutamate transporter 3 (VGLUT3). To date, hiPSC-NPCs have been reported to terminally differentiate into neurons such as VGLUT1positive glutamatergic neurons and glutamate decarboxylase 67 (GAD67)-positive GABAergic neurons [8-11]. However, there have been a few reports on the induction of excitatory neurons other than VGLUT1-positive glutamatergic neurons. Although it has been reported that synapses themselves change from immature to mature states as neurons mature, few papers have clearly demonstrated when human stem cell-derived neurons change to mature synaptic structures [12]. Several reports have shown that hiPSC-derived neurons (hiPSC-neurons) have neurophysiological activity in vitro [13], but only a few reports have confirmed the localization of mature synaptic markers such as the PSD-95 protein. Moreover, most previous studies used mainly the direct induction method without a continuous proliferation process for NPCs, and the types of neurons that differentiate from in vitro expanded NPCs (which can advantageously be induced into a relatively homogeneous neuronal population on a large scale) and the extent of synapserelated molecule maturity remain unclear $[8,14]$.

In this study, we differentiated hiPSC-NPCs that were established using dual SMAD inhibition and expanded them by neurosphere culture with epidermal growth factor (EGF), fibroblast growth factor 2 (FGF2), and leukemia inhibitory factor (LIF). We examined the expression profiles of neuronal lineage, presynaptic, and postsynaptic markers. We hypothesized that some time would be required to allow for the expression and localization of PSD-95 in human-derived cells. To capture the changes in the synaptic structure at an earlier stage, we evaluated a marker called drebrin. There are two subtypes of drebrin in mammals, including in humans, drebrin $\mathrm{A}$ and drebrin $\mathrm{E}[15,16]$. This marker is an actin-binding protein that replaces drebrin $\mathrm{E}$ with drebrin $\mathrm{A}$ by alternative splicing during neuronal maturation $[17,18]$, and we also evaluated the effect of nerve growth factor (NGF) on the synaptic maturation of hiPSC-NPCs. NGF is produced in various parts of the CNS, and its action is particularly important for the survival of cholinergic nerves in the basal forebrain and has been reported to be involved in dendrite formation, at least in cholinergic neurons [1921]. Thus, we expected that the presence or absence of NGF would alter synaptogenesis and differentiation maturation into cholinergic nerve cells. We believe that this study will clarify the in vitro process of neurogenesis and synapse formation of hiPSC-NPCs and provide useful information about the potential for future in vitro neuronal research and pharmacological testing applications using these hiPSC-neurons.

\section{Materials and methods}

\section{Ethics statement}

This study was conducted in accordance with the principles of the Declaration of Helsinki, and the use of hiPSCs and human fetal neural tissue-derived neural stem/ progenitor cells (hN-NSPCs) was approved by the ethics committee of Osaka National Hospital (\#110, 120, and 146).

\section{Cell lines}

We used two hiPSC lines, 1210B2 and 1201C1 [22, 23], which were established from peripheral mononuclear blood cells using an integration-free reprogramming method and a feeder-free protocol (1210B2) [24] or SNL 
feeder cells (1201C1) [25]. Both lines were induced into NPCs using the dual SMAD inhibition method with dorsomorphin (FUJIFILM Wako Pure Chemical Corporation, Osaka, Japan) or LDN-193189 (Axon Medchem, Groningen, Netherlands) plus SB431542 (Sigma-Aldrich, St. Louis, MO, USA), as previously described [22, 23]. These hiPSC-NPCs were propagated using the neurosphere method in DMEM/Ham's F-12 (DMEM/F12; FUJIFILM Wako Pure Chemical Corporation) supplemented with EGF (20 ng/mL; PeproTech, Rocky Hill, NJ, USA), FGF2 (20 ng/mL; PeproTech), LIF (10 ng/mL; Millipore, Billerica, MA, USA), B27 Supplement (B27, 2\%; Thermo Fisher Scientific, Waltham, MA, USA), and heparin (1/1000 dilution; AY Pharmaceuticals, Tokyo, Japan) [26]. The medium was changed every $3-5$ days. The cells were passaged every 10-12 days using TrypLE Select CTS (Thermo Fisher Scientific) at $37^{\circ} \mathrm{C}$ for $5 \mathrm{~min}$ for single-cell dissociation and then resuspended in DMEM/ Ham's F-12 medium containing B27 Supplement, heparin, LIF, EGF, and FGF2, at a density of $1 \times 10^{5}$ cells $/ \mathrm{mL}$ [27].

Frozen dissociated primary hippocampal neurons of rats (SKY neurons; AlzMed, Tokyo, Japan) were cultured in PLO-coated dishes for 21 days according to the manufacturer's instructions and used as controls for immunostaining and electrophysiological analysis $[28,29]$.

\section{In vitro neuronal differentiation}

In vitro neuronal induction was performed as shown in Fig. 1a. Neurospheres were dissociated into single cells and seeded onto poly-L-ornithine $(0.1 \mathrm{mg} / \mathrm{mL}$; SigmaAldrich)-coated 96-well plates (ibidi, Gräfelfing, Germany) at a density of $2.25 \times 10^{5}$ cells $/ \mathrm{cm}^{2}$ in Neurobasal Plus Medium (Thermo Fisher Scientific) containing B27 Plus Supplement (B27 Plus, 2\%; Thermo Fisher Scientific), GlutaMAX (0.5 mM; Thermo Fisher Scientific), gamma-secretase inhibitor, DAPT $(10 \mu \mathrm{M}$; Abcam, Cambridge, UK), and brain-derived neurotrophic factor (BDNF, $20 \mathrm{ng} / \mathrm{mL}$; PeproTech). The Rho-associated coiled-coil forming kinase inhibitor Y-27632 (Y, $10 \mu \mathrm{M}$; FUJIFILM Wako Pure Chemical Corporation) was added only on the 1st day. One day later (day 1 of differentiation), the medium was exchanged (half of the volume), and half of the medium was exchanged every 3-4 days thereafter. The culture was continued until day 49 of differentiation in the presence or absence of nerve growth factor (10 ng/mL; PeproTech).

\section{Quantitative reverse transcription-polymerase chain reaction (qRT-PCR)}

The total RNA was separated using QIAzol Lysis Reagent (QIAGEN, Hilden, Germany), and cDNA was synthesized using the PrimeScript RT Reagent Kit (Takara
Bio Inc., Shiga, Japan) according to the manufacturer's instructions. qRT-PCR analysis was performed using gene-specific primers (Additional file 1: Table 1), PowerUp SYBR Green Master Mix (Thermo Fisher Scientific), and the 7300 Real-Time PCR System (Applied Biosystems, Foster City, California, USA), and the relative expression levels were calculated by the comparative threshold cycle $(\mathrm{Ct})$ method.

Four control RNAs were used: two from hN-NSPCs (oh-NSC-3-fb and oh-NSC-8-sp) [26], human brain cerebellum total RNA and human brain cerebrum total RNA (Takara Bio Inc., CA, USA).

\section{Immunocytochemical staining}

The cells were fixed with $4 \%$ paraformaldehyde phosphate buffer solution (FUJIFILM Wako Pure Chemical Corporation) at room temperature, washed three times with phosphate-buffered saline (PBS), and then blocked with a blocking solution (10\% normal goat serum, $0.1 \%$ Triton X-100, PBS) for 30-60 min at room temperature. The blocking solution was then replaced with the primary antibody solution shown in Additional file 2: Table 2. After incubation at $4{ }^{\circ} \mathrm{C}$ overnight, the primary antibody solution was removed, and the cells were washed three times with PBS. Next, the cells were incubated with an AlexaFluor 488-conjugated goat anti-mouse IgG antibody (Thermo Fisher Scientific), an AlexaFluor 568-conjugated goat anti-rabbit IgG antibody (Thermo Fisher Scientific), and DAPI (Dojindo, Kumamoto, Japan) for 1 $h$ at room temperature. The stained samples were then evaluated with a confocal laser scanning microscope (LSM700; Carl Zeiss, Hallbergmoos, Germany).

Under each condition, the number of ELAVL3/4-positive cells among 150 or more DAPI-positive cells was determined. In addition, ten visual fields were examined for each staining condition, and the proportions of cells that were positive for VGLUT1, VGLUT2, tyrosine hydroxylase $(\mathrm{TH})$, tryptophan hydroxylase 2 (TPH2), vesicular acetylcholine transporter (VAChT), choline acetyltransferase (ChAT), and glial fibrillary acidic protein (GFAP) were determined.

\section{Western blot analysis}

The cells were lysed with cell lysis buffer (2\% SDS, $50 \mathrm{mM}$ Tris- $\mathrm{HCl} \mathrm{pH} 8.0,150 \mathrm{mM} \mathrm{NaCl}$ ), and $30 \mu \mathrm{g}$ of protein from each sample was separated using sodium dodecyl sulfate-polyacrylamide gel electrophoresis (SDS-PAGE) and then electrophoretically transferred to polyvinylidene difluoride membranes (GE healthcare, Chicago, IL, USA). The membranes were blocked with $2 \%$ ECL Prime Blocking Reagent (cat. RPN418, GE healthcare) and incubated at $4{ }^{\circ} \mathrm{C}$ overnight with the primary antibody solution (Additional file 3: Table 3). After washing 
a
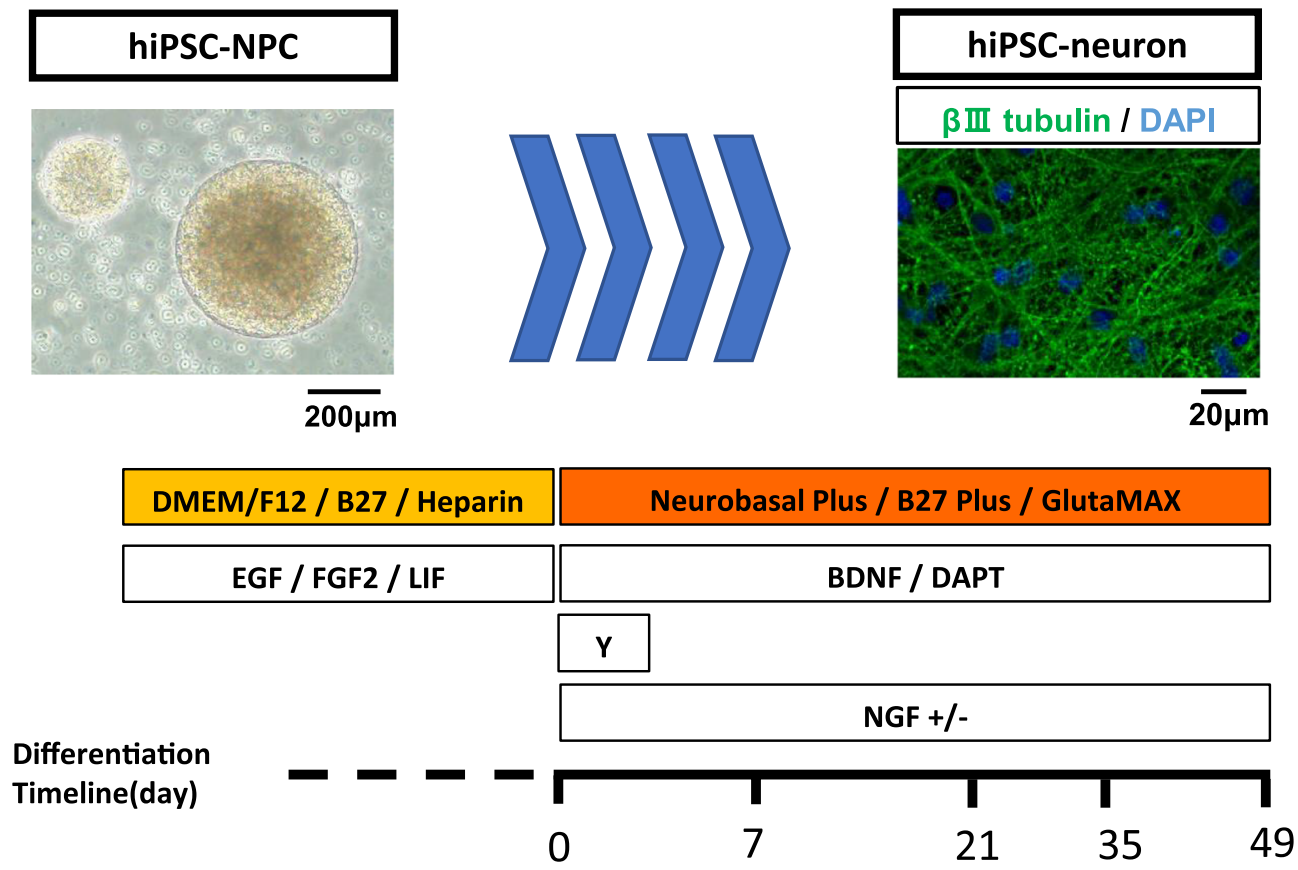

b

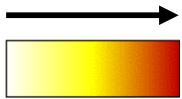

Resional specificity markers

FOXG1 OTX2 EN1 GBX2 HOXB4 PAX6 PAX7 NKX2.1 NKX2.2 NKX6.1 EMX2

\begin{tabular}{|c|c|c|c|c|c|c|c|c|c|c|c|}
\hline oh-NSC-3-fb & 4.32 & 7.07 & UD & 9.64 & UD & 8.18 & UD & 15.55 & UD & UD & 12.46 \\
\hline h-NSC-8-sp & 6.82 & 17.56 & 8.93 & 5.63 & 10.16 & 7.50 & UD & 6.56 & 10.99 & 9.91 & UD \\
\hline 1210B2 & UD & 11.93 & 8.74 & 5.61 & UD & 8.10 & 10.74 & UD & UD & 9.44 & UD \\
\hline $1201 \mathrm{C1}$ & 11.21 & 11.00 & 15.11 & 5.88 & UD & 7.49 & UD & UD & UD & 6.07 & 10.80 \\
\hline
\end{tabular}

Fig. 1 Procedure for the neuronal differentiation and characterization of hiPSC-NPCs. a Procedure for the neuronal differentiation of hiPSC-NPCs. The phase-contrast image shows the neurosphere of the hiPSC-NPCs before differentiation, and the immunofluorescence image shows neurons derived from hiPSC-NPCs (green: $\beta$ III-tubulin, blue: DAPI). Scale bars: $200 \mu \mathrm{m}$ and $20 \mu \mathrm{m}$. b Gene expression of hiPSC-NPCs (1210B2 and 1201C1) and human neural tissue-derived NSPCs (oh-NSC-3-fb and oh-NSC-8-sp) before differentiation. The $\triangle C$ t values are shown. Red: high expression. UD undetermined

with Tris-buffered saline with Tween 20 (TBS-T), the membranes were incubated with secondary antibodies against rabbit or mouse horseradish peroxidase-conjugated immunoglobulin G (IgG, Cell Signaling Technology). Protein bands were detected by ECL Prime Western Blotting Detection Reagents (cat. RPN2232; GE healthcare), and images were obtained with the LAS4000 (Fujifilm, Tokyo, Japan). For reprobing, the membrane was stripped with stripping buffer (cat. T7135A; Takara Bio), blocked with 2\% ECL Prime Blocking Reagent, and reprobed with different primary and secondary antibodies. Blotting with an anti- $\beta$-actin antibody (Additional file 3: Table 3) was used as the control. Quantification was performed with ImageJ software (Fiji package; National Institutes of Health, Bethesda, MD, USA). Ten micrograms of rat cerebrum tissue lysate (Takara Bio) was loaded and used as the control. 
(See figure on next page.)

Fig. 2 Gene expression levels of pre- and postsynaptic markers during the neuronal differentiation of hiPSC-NPCs. Dot plots and boxplots showing the expression of a presynaptic markers (synaptophysin and synapsin I) and $\mathbf{b}$ postsynaptic markers (drebrin A+E, drebrin A, PSD-95, NR1, GLUR2) during neuronal differentiation. Statistical analysis was performed using ANOVA with the post hoc Tukey-Kramer method $\left({ }^{* * *} p<0.001,{ }^{* *} p<0.01\right.$, $\left.{ }^{*} p<0.05\right)$. UD undetermined

\section{Microelectrode array (MEA) recording}

MEA recording was performed using the 60-channel MEA2100 system (Multi Chanel Systems, Reutlingen, Germany). The hiPSC-NPCs were differentiated into 6-well plates using the same methods as above, and hiPSC-neurons on day 49 were prepared for MEA recording. Spontaneous activity measurements were performed at $37^{\circ} \mathrm{C}$ at a sampling rate of $20 \mathrm{kHz}$ and filtered using a $200-3000 \mathrm{~Hz}$ band-pass filter. After measurement in differentiation medium without added glutamate, the whole volume of the medium was changed to differentiation medium with $100 \mu \mathrm{M}$ glutamate, and the measurement was performed again. The obtained waveforms were analyzed using NeuroExplorer Version 5.306 (Nex Technologies, Madison, Alabama, USA). The standard deviation $(\mathrm{SD})$ was calculated, and $-6.0 \times \mathrm{SD}$ of the baseline electrode noise was set as the negative threshold [30]. The negative peaks that exceeded the threshold were calculated as spikes and the presence of bursts was also analyzed by MaxInterval method [31]. SKY neurons at 21 days in vitro were used as a positive control, and wells with no cells were seeded as a negative control.

\section{Statistical analysis}

The $\Delta \mathrm{Ct}$ value was calculated based on normalization to glyceraldehyde-3-phosphate dehydrogenase (GAPDH) for each condition. For each condition, the average $\Delta \mathrm{Ct}$ value was calculated, and a box plot was created. The $\Delta \mathrm{Ct}$ value of drebrin $A$ based on drebrin $A+E$ was calculated to determine the fractional ratio of drebrin. Student's $t$-tests and one-way analysis of variance (ANOVA) were used for $\triangle \mathrm{Ct}$ values obtained under each qRT-PCR condition. When a significant difference was found in the ANOVA tests, multiple comparison tests using the Tukey-Kramer method were performed as post hoc analysis. A p-value $<0.05$ was considered to be statistically significant. Data that could not be reproduced were treated as missing values.

\section{Multicenter validation study}

Cells from the 1210B2 line were transported in a cryopreserved form and subsequently used. Then, the cells were cultured in DMEM/Ham's F-12 medium containing B27 Supplement, heparin, LIF, EGF, and FGF2 for 10 days and dispersed into single cells with Neuron Dissociation Solutions S (FUJIFILM Wako Pure Chemical Corporation) according to the manufacturer's instructions. Then, the hiPSC-NPCs were differentiated into neurons using the same induction method as that used in the absence of NGF described above. Total RNA was extracted before induction and at 49 days after neuronal differentiation using an RNeasy plus micro kit (QIAGEN), and RNA extraction samples from each facility were collected. Then, cDNA synthesis was performed using Prime Script RT (Takara Bio Inc.). qRT-PCR analysis was performed as described above using the comparative threshold cycle (Ct) method. The $\triangle \mathrm{Ct}$ values calibrated by normalization to GAPDH were determined for each gene, and the high and low expression levels were analyzed using a heat map to examine the robustness of our differentiation induction method.

\section{Results}

Expression of the ventral hindbrain marker in hiPSC-NPCs

We first characterized the hiPSC-NPCs just before neuronal differentiation. Regional markers along the anterior/posterior and dorsal/ventral axes of both the 1210B2 and $1201 \mathrm{C} 1$ cell lines were examined using qRT-PCR. Human fetal forebrain-derived NSPCs (oh-NSC-3-fb) and fetal spinal cord-derived NSPCs (oh-NSC-8-sp) were used as controls. Both hiPSC-NPC lines expressed the ventral hindbrain markers GBX2 (mid/hindbrain) and NKX6-1 at relatively high levels (Fig. 1b), showing that the two hiPSC-NPCs have hindbrain regional specificity. Focusing on the expression of other localization markers, FOXG1, a forebrain marker, was highly expressed in oh$\mathrm{NSC}-3$-fb cells. OTX2, a forebrain and midbrain marker, was also relatively expressed in oh-NSC-3-fb cells, while these markers were not expressed well in the hiPS-NPCs used in this study. $\mathrm{HOXB4}$, a marker of spinal cord localization, was expressed in oh-NSC-8-sp cells but not in the oh-NSC-3-fb, 1210B2 or 1201C1 cell lines.

\section{Gene expression of pre- and postsynaptic markers increased during neuronal differentiation}

mRNA transcription analysis by qRT-PCR demonstrated that the expression levels of synaptophysin (SYP) and synapsin I (SYN1) (presynaptic markers) as well as drebrin $A$, PSD-95, NR1 and GLUR2 (postsynaptic markers) significantly increased in the two hiPSC-NPC lines as neuronal differentiation progressed (Fig. 2a, b). Moreover, the expression level of drebrin $A+E$ did not change during 


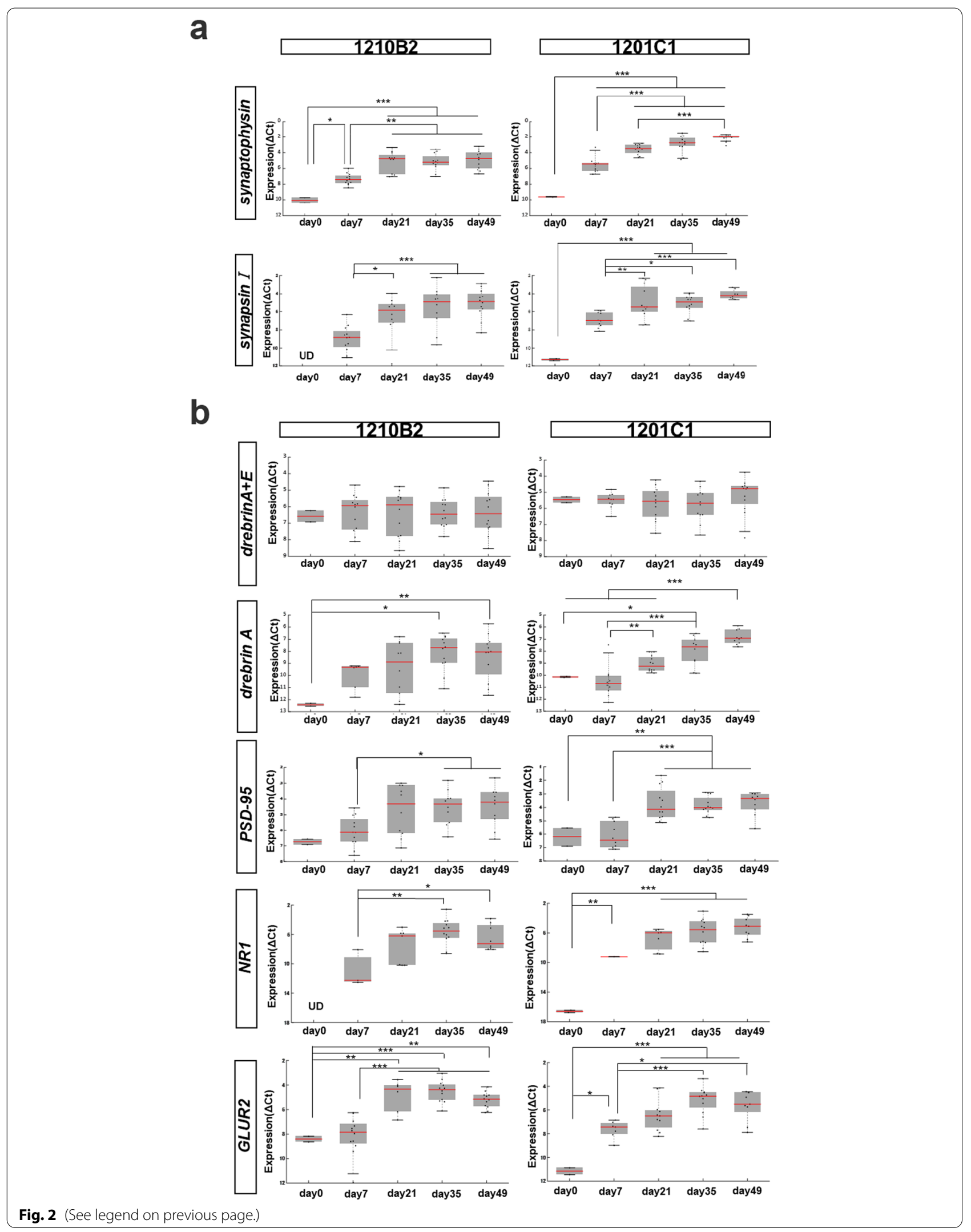


differentiation (Fig. 2b). We also examined the effect of NGF on the gene expression of drebrin $A+E$, drebrin $A$, and $S Y P$; however, there were no significant differences in the expression levels of the three genes between the groups with and without NGF (Additional file 4: Fig. S1). These findings indicated that hiPSC-NPC terminal differentiation was accompanied by the expression of pre- and postsynaptic marker genes and the isoform conversion from drebrin $E$ to drebrin $A$ and that NGF had no significant effect on the expression of pre- and postsynaptic marker genes or on the isoform conversion of drebrin.

\section{Protein expression of pre- and postsynaptic markers in hiPSC-neurons on day $\mathbf{4 9}$ of differentiation}

Immunofluorescence microscopy showed marked levels of BIII-tubulin-positive axonal growth in hiPSC-neurons during the differentiation period (Fig. 3 and Additional file 4: Fig. S2). Both microtubule-associated protein 2 (MAP2)-positive dendrites and pan-drebrin (drebrin $\mathrm{A}+\mathrm{E}$ )-positive dendrites were obviously expressed during the differentiation period (Fig. 3 and Additional file 4: Fig. S2). A presynaptic marker, synaptophysin (SYP), was clearly expressed on day 21 of differentiation, and its expression level increased over time. However, the expression of a postsynaptic marker, drebrin A, was weak, even after day 35. Additionally, the expression of another postsynaptic marker, PSD-95, was poor even on day 49 in comparison with sufficient expression on day 21 SKY neurons (Fig. 3, Additional file 4: Figs. S2 and S3). As with transcript expression, NGF did not show a significant effect on the expression of synapse-related proteins (Additional file 4: Fig. S4). These findings suggested that the hiPSC-neurons expressed neuronal markers and synapse-related proteins at the protein level. However, the expression levels of these proteins were different; the expression of postsynaptic markers was relatively modest and lower than that of presynaptic markers.

The expression levels of pre- and postsynaptic markers were also validated using Western blotting (Additional file 4: Fig. S5 and Fig. 4). Presynaptic markers, BIIItubulin and synaptophysin, were both expressed much higher in hiPSC-neurons (day 49) than hiPSC-NPCs (day 0 ); however, their relative expression levels were lower than those in rat cerebral tissues (Additional file 4: Figs. S5, S6). The pan-drebrin was expressed equally in both hiPSC-NPCs (day 0) and hiPSC-neurons (day 49), and semiquantitative analysis showed that their expression levels were almost stable and slightly higher than those in rat cerebral tissues, which was consistent with the results of mRNA analysis (Additional file 4: Fig. S6). Drebrin A was significantly detected only in hiPSC-neurons (day 49); however, its relative expression level was much lower than that in rat cerebral tissues (Fig. 4 and Additional file 4: Fig. S6). Other postsynaptic markers, including PSD-95, GluA1 and GluA2, were expressed at detectable levels only in hiPSC-neurons, but their expression levels were also lower than those in rat cerebral tissues (Fig. 4 and Additional file 4: Fig. S6). NR1 was expressed in hiPSC-NPCs and increased after neuronal differentiation, but it remained at approximately one tenth of the level in the normal rat cerebrum. These findings indicated that postsynaptic markers were surely expressed in hiPSC-neurons at the protein level, in accordance with transcript analysis.

\section{Evaluation of electrophysiological activity of hiPSC-neurons}

To examine the neurophysiological maturation of hiPSCneurons, we analyzed the electrophysiological activity of hiPSC-neurons on day 49 of differentiation using SKY neurons as positive controls. In the positive control, spontaneous spikes were apparently detected in the glutamate-free medium at 21 days, and burst discharges were identified at several measurement points after the addition of $100 \mu \mathrm{M}$ glutamate, which suggests neurophysiological maturation, although it did not reach a level that showed a significant increase in the total spike frequency (Fig. 5). On the other hand, neither spontaneous spikes nor the response to glutamate stimulation were observed in hiPSC-neurons on day 49 (Fig. 5). These findings indicated that the neurophysiological maturation of hiPSC-neurons was not sufficiently developed on day 49 of differentiation and that they stayed in their immature stage.

\section{Comparison of pre- and postsynaptic marker gene expression between adult human brain samples and hiPSC-neurons}

On day 49 of differentiation, the gene expression (normalized to GAPDH) of the presynaptic marker $S Y P$ in $1201 \mathrm{C} 1$ (but not in 1210B2) cells was similar to that in commercially available adult human cerebellum and cerebrum samples regardless of whether NGF was added (Fig. 6a). Another presynaptic marker, SYN1, was expressed in both cell lines at the same level as that in adult brain tissue samples (Fig. 6b). Since there was no significant change in drebrin $A+E$ mRNA expression over time in our culture and previous reports have shown alternative splicing from drebrin $E$ to drebrin $A$, we hypothesized that changes in the drebrin fraction would alter the expression of postsynaptic markers. The expression ratio of drebrin $A$ to drebrin $A+E$ in both the 1210B2 and $1201 \mathrm{C} 1$ cell lines tended to be lower than that in the cerebellum samples and significantly lower than that in the cerebrum samples (Fig. 6c). In contrast to drebrin A, PSD-95 expression was the same as that in 


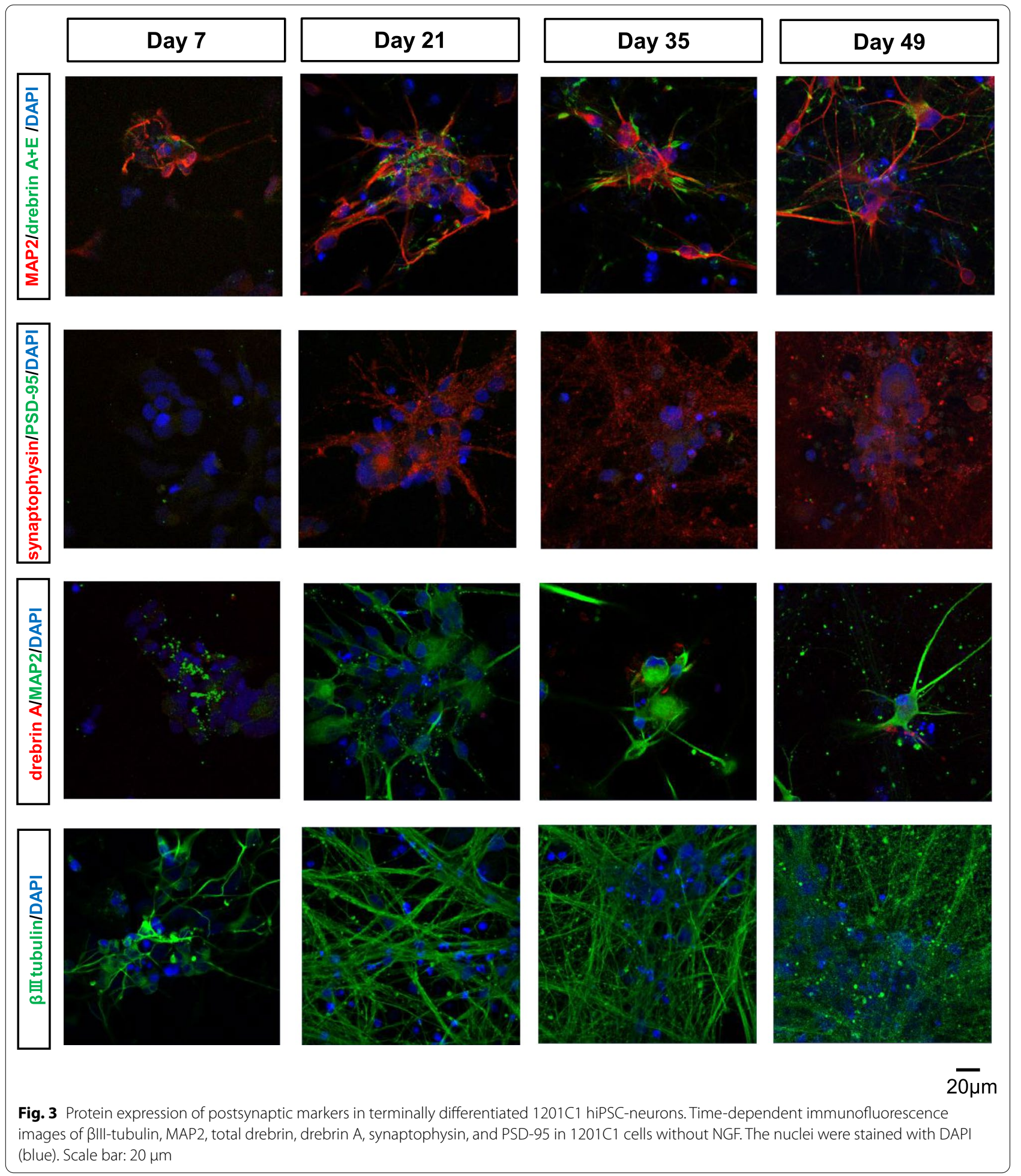

the adult samples (Fig. 6d). These findings indicate that although hiPSC-neurons generally express pre- and postsynaptic markers at the transcriptional level, the expression levels vary depending on the marker. In particular, drebrin $A$ expression does not reach the level of that in adult cerebral tissue. 

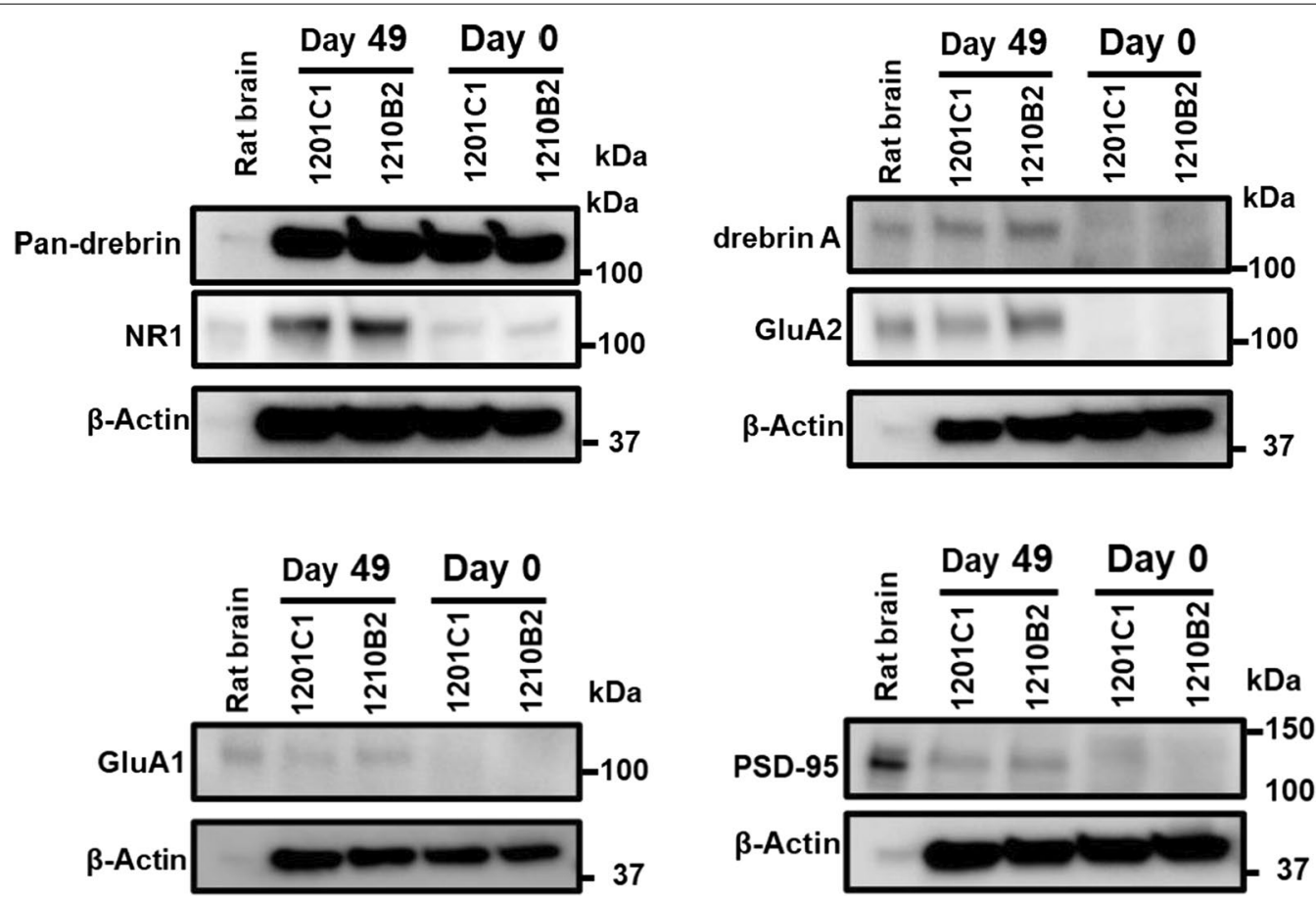

Fig. 4 Western blot analysis of postsynaptic proteins in hiPSC neurons on day 0, day 49 and rat cerebrum tissue lysate. For the rat cerebrum tissue sample, $10 \mu \mathrm{g}$ of protein per well was used, and for the other samples, $30 \mu \mathrm{g}$ of protein per well was used for analysis

\section{Phenotypes of hiPSC-derived neurons after 49 days of differentiation}

The number of neurons determined by the ratio of ELAVL3/4-positive cells on day 49 reached $80 \%$ or more of the population in two cell lines (1210B2 cell line: NGF+, 88.8\%; NGF-, $82.9 \%$ and $1201 \mathrm{C} 1$ cell line: NGF+, 80.2\%; NGF-, 82.8\%). Most neurons (> 80\%) were VGLUT2-positive glutamatergic neurons (Fig. 7a, Table 1). The number of neurons that were positive for VGLUT1 (another glutamatergic neuronal marker), TH (dopaminergic neuron marker), VAChT, or ChAT (cholinergic neuron marker) was very small (Fig. 7a, Table 1). Additionally, TPH2 (a serotoninergic marker) was not detected in any neurons (Table 1). While few GFAP-positive glial cells were found, many fibers were observed (Fig. 7a). The differential expression patterns of VGLUT1 and VGLUT2 on glutamatergic neurons were confirmed by qRT-PCR analysis (Fig. 7b, c); the expression of VGLUT2 was higher than that in adult human brain tissue samples (Fig. 7c). This result was in contrast to that of VGLUT1 expression (Fig. 7b). These findings showed that hiPSC-NPCs predominantly differentiated into VGLUT2-positive glutamatergic neurons in our culture system.

\section{Multi-institutional validation of the reproducibility of the neural differentiation method}

To evaluate the efficacy and robustness of the differentiation method developed here, the 1210B2 cell line was evaluated at multiple facilities under non-NGF cell culture medium conditions. On days 0 and 49, we performed qRT-PCR analysis to compare the cells that were differentiated at multiple sites (Fig. 8). Similar gene expression patterns were observed in both hiPSC-NPCs on day 0 and hiPSC-neurons on day 49 regardless of the facility at which they were differentiated (Fig. 8). Undifferentiated markers such as SOX1, PAX6, and NES were expressed at low levels, reflecting differentiation. The expression of localization markers such as EN1 and $G B X 2$ tended to be lower than that before differentiation. Focusing on the markers of glutamatergic neurons, the expression of VGLUT1 was not reproducible, but VGLUT2 expression increased as the cells differentiated. This result was consistent with the immunostaining images presented earlier, which showed a VGLUT2-dominated cell population. Although only a few cells were observed in the stained images, the expression levels of markers of GABAergic neurons, cholinergic neurons, dopaminergic neurons, and serotonergic neurons as well as glial cells were increased, which suggested that the induction of these cell types was advancing. The expression levels of the presynaptic markers SYP and SYN1, the 
a

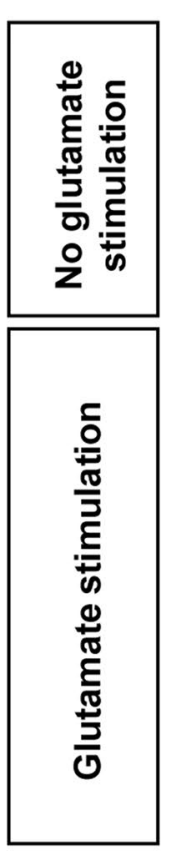

Positive Control
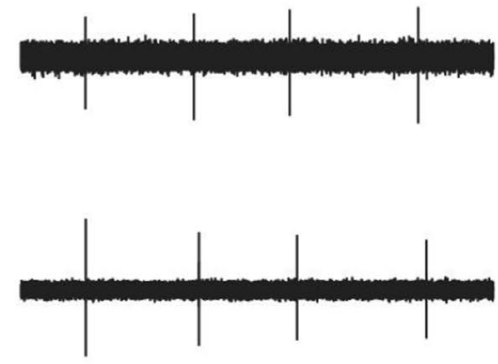

$*$

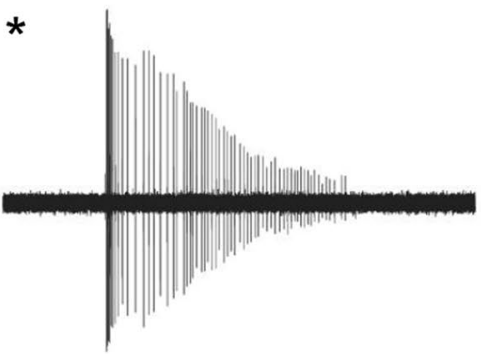

1201C1 day 49
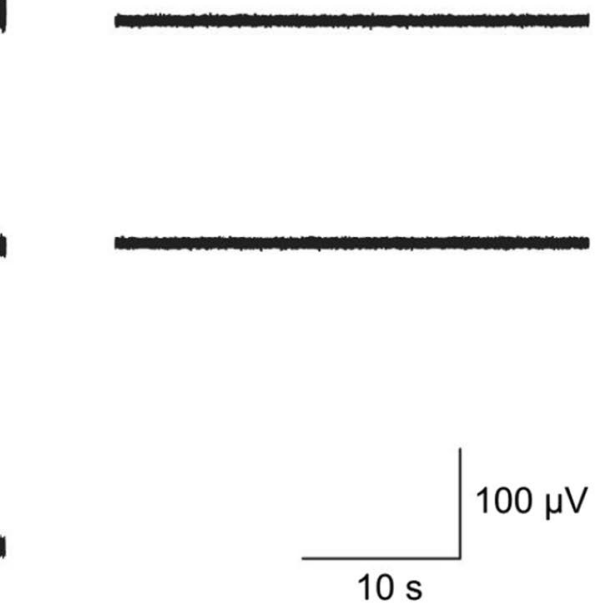

\section{b}

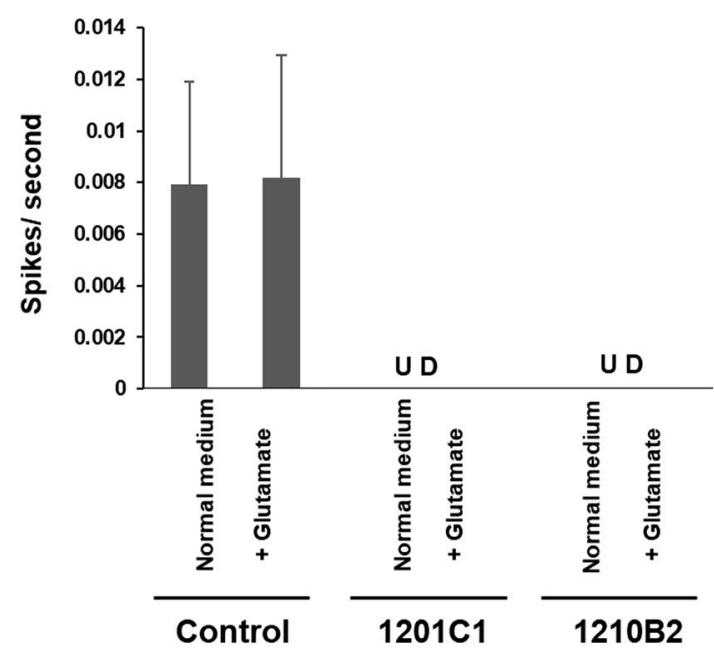

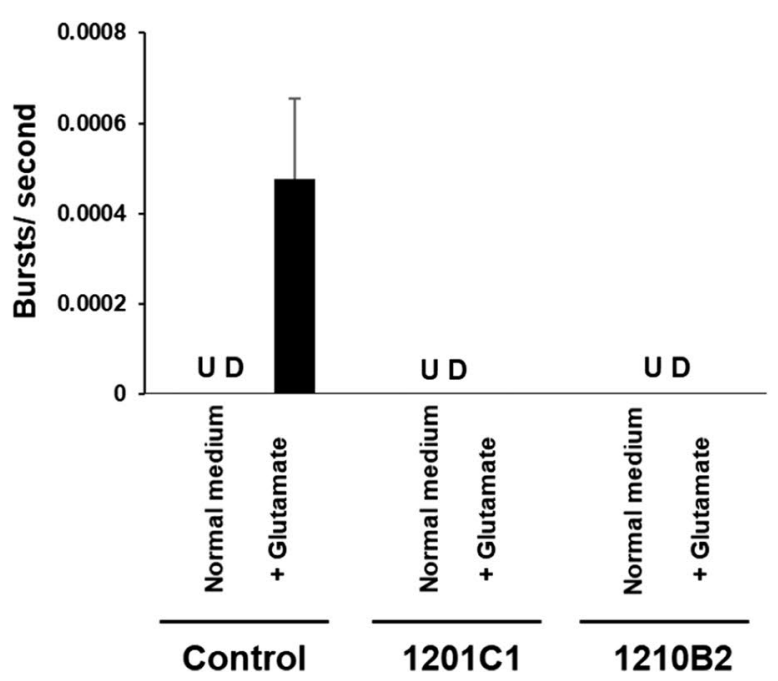

Fig. 5 Microelectrode array recording results. a Examples of electrophysiological activity are presented. The burst firing seen in the positive control during glutamate stimulation is additionally shown $\left(^{*}\right) . \mathbf{b}, \mathbf{c}$ The graph shows the number of spikes and bursts per second. The bars show the mean, and the error bars show the standard error. Spikes and bursts were detected in the positive control, but no significant spike was detected in the hiPSC-neurons. UD undetermined

excitatory postsynaptic markers $P S D-95$ and drebrin, and the inhibitory postsynaptic marker gephyrin as well as those of AMPA and NMDA receptor markers were also confirmed, which suggested progression in the direction of synapse formation. Moreover, approximately $80 \%$
(75-87\%) of the cell population was ELAVL3/4-positive on day 49 at all facilities. 


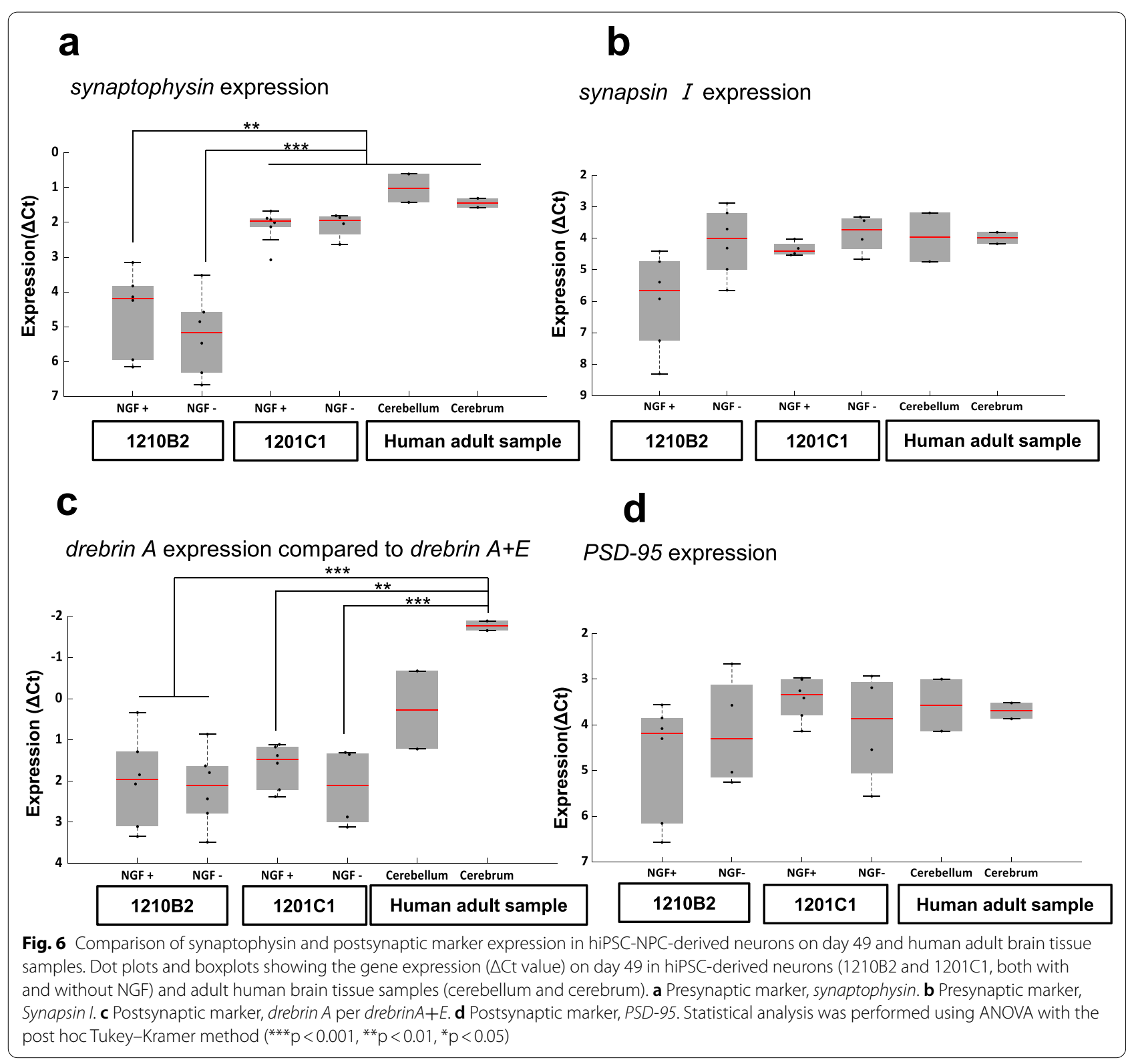

\section{Discussion}

Human iPSC-NPCs differentiate into mature neurons expressing neuronal markers and synapse-related proteins, but the expression of postsynaptic markers is incomplete

In this study, we confirmed sufficient maturation of hiPSC-neurons at the level of neuronal phenotypic markers after 49 days of culture. The presynaptic markers SYP and SYN1 were generally well expressed at the mRNA level by day 35 , which was generally consistent with the immunostaining images.

However, the postsynaptic markers differed from presynaptic markers. The drebrin $\mathrm{E}$ protein was found to be expressed early, whereas the other postsynaptic markers were detected at the level of mRNA transcription, but their protein expression and localization to dendritic spines were insufficient in immunostaining images.

Western blot analysis showed that the protein expression of both presynaptic and postsynaptic markers was equally increased in the day 49 hiPSC-neuron sample compared to the day 0 hiPSC-neuron sample, although the expression was lower compared to the rat brain tissue sample. This suggests that the differences in immunostaining results of pre- and postsynaptic proteins cannot be explained by protein expression alone. The differences in the localization of synaptic proteins may be 
a
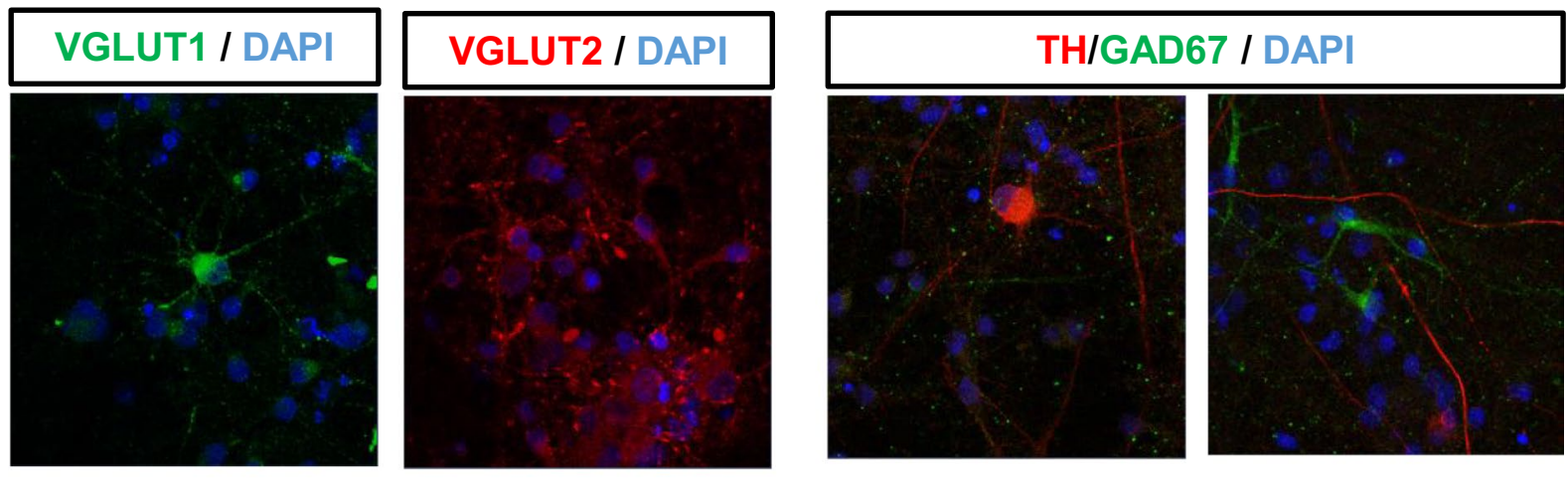

$20 \mu \mathrm{m}$

$20 \mu \mathrm{m}$

$20 \mu \mathrm{m}$

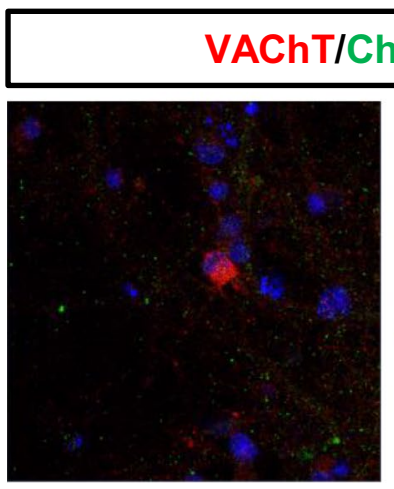

\section{AT I DAPI}
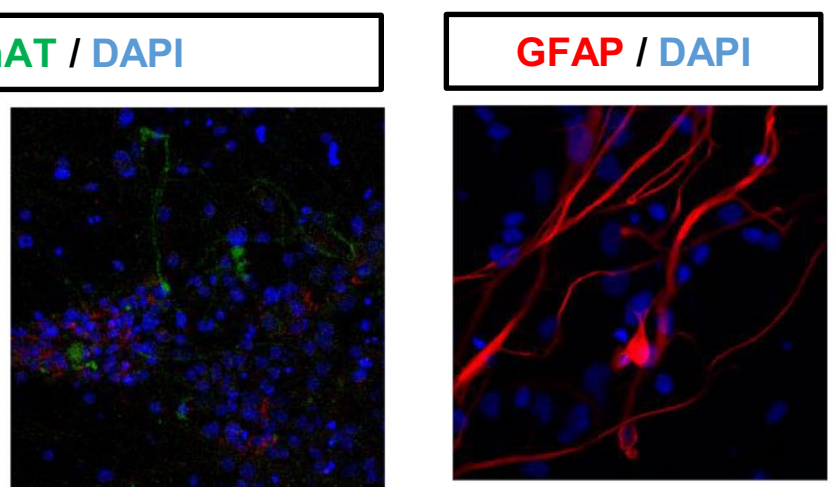

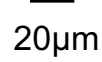

$20 \mu \mathrm{m}$

b
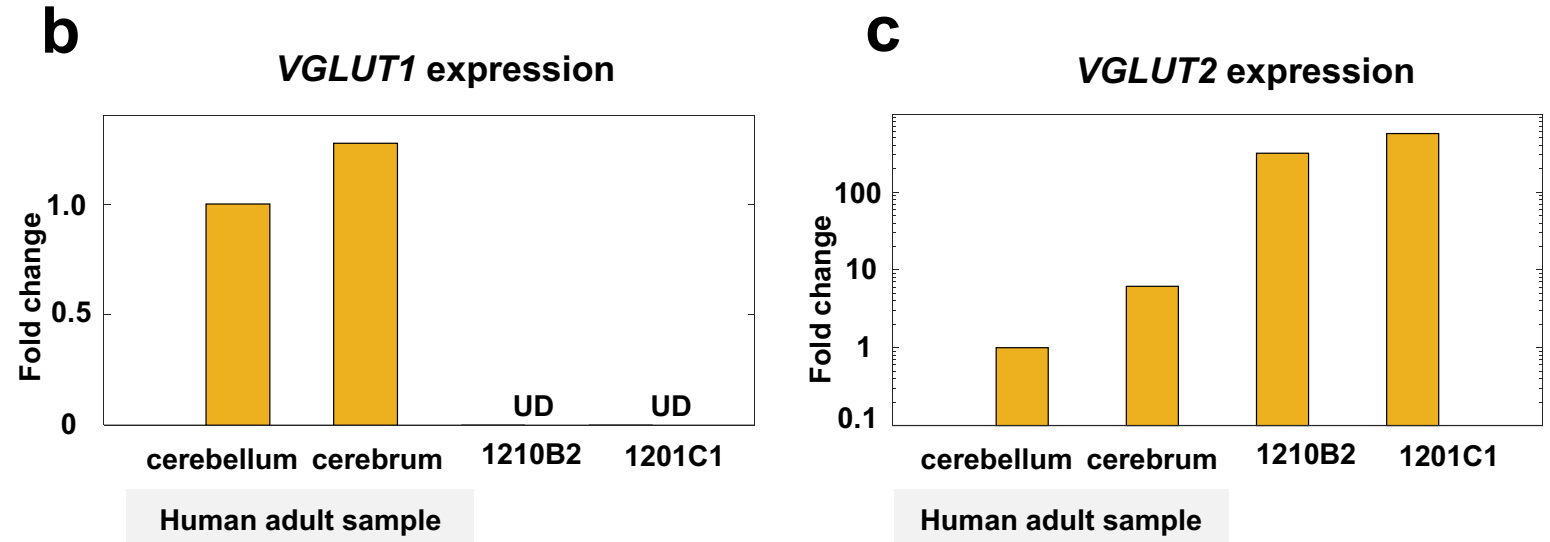

Fig. 7 Phenotypic analysis of hiPSC-neurons on day 49 of differentiation. a Immunofluorescence analysis of glutamatergic neurons (VGLUT1 and VGLUT2), dopaminergic neurons (TH), GABAergic neurons (GAD67), cholinergic neurons (VAChT and ChAT), and glial cells (GFAP) on day 49 of differentiation in 1210B2 cells. The nuclei were stained with DAPI (blue). Scale bar: $20 \mu \mathrm{m}$. b, c Comparison of the gene expression levels of VGLUT1 (b) and VGLUT2 (c) in hiPSC-derived neurons (1210B2 and 1201C1, both without NGF) on day 49 and in adult human samples (cerebellum and cerebrum). UD undetermined

reflected in the differences in immunostaining results. Functional analysis with MEA recording could not detect significant electrophysiological activity of hiPSC-neurons on day 49. This finding is consistent with previous reports showing that it takes a long time to detect electrophysiological activity in human stem cell-derived neurons 
Table 1 Neuronal subtypes analysis of hiPSC-NSPC-derived neurons after 49 days of differentiation

\begin{tabular}{|c|c|c|c|c|c|c|c|c|}
\hline \multirow[t]{3}{*}{ hiPSC neurons } & \multirow[t]{3}{*}{ NGF } & \multicolumn{7}{|c|}{ Type of cells } \\
\hline & & \multicolumn{2}{|c|}{ Glutamatergic nerve } & \multirow{2}{*}{$\begin{array}{l}\text { Dopaminergic } \\
\text { neuron } \\
\text { TH positve }\end{array}$} & \multirow{2}{*}{$\begin{array}{l}\text { Serotonergic } \\
\text { neuron } \\
\text { TPH2 positve }\end{array}$} & \multicolumn{2}{|c|}{ Cholinergic neuron } & \multirow{2}{*}{$\begin{array}{l}\text { Glial cells } \\
\text { GFAP positive }\end{array}$} \\
\hline & & $\begin{array}{l}\text { VGLUT1 } \\
\text { positive }\end{array}$ & VGLUT2 positive & & & VAChT positve & ChAT positve & \\
\hline \multirow[t]{2}{*}{ 1210B2 } & + & + & +++ & + & - & + & + & + \\
\hline & - & + & +++ & + & - & + & + & + \\
\hline \multirow[t]{2}{*}{$1201 C 1$} & + & + & +++ & + & - & + & + & + \\
\hline & - & + & +++ & + & - & + & + & + \\
\hline
\end{tabular}

The evaluation of immunofluorescence images are shown (+++:>80\%; ++: 10-80\%; +: 1-10\%; -: none)

$[13,32]$. Taken together, these results suggest that the maturation of synaptic structures of hiPSC-neurons was incomplete and that hiPSC-neurons remained immature in their neurophysiological features after 49 days of differentiation.

There are two forms of drebrin, the juvenile form of drebrin $\mathrm{E}$ and the mature form of drebrin $\mathrm{A}$, which are generated by alternative splicing from a single gene [17]. During neuronal development, drebrin E is present in growth cones and is involved in axon extension. As neurons mature, drebrin $\mathrm{E}$ disappears, and drebrin A appears, accumulating in the postsynaptic region and promoting the formation of dendritic spines [17]. Since drebrin A promotes the accumulation of PSD-95, the conversion of drebrin $E$ to drebrin $A$ expression is thought to be a useful marker of postsynaptic immaturity and maturation in stages without PSD-95 expression [18, 33]. Focusing on its expression, drebrin $A$ was increased for up to 35 days in 1210B2-derived neurons and for up to 49 days in 1201C1-derived neurons. Changes in drebrin A mRNA expression were consistent with the trend of changes in the expression levels of other postsynaptic markers, PSD-95, GluR1, and NR1. These findings suggest that our method requires at least 35 days of incubation for dendritic maturation at the mRNA level. However, even at week 7 , the protein expression and localization of postsynaptic maturation markers were poor. These results suggest that when examining synaptic maturation, we should be aware that the construction of postsynaptic structures is slower than the differentiation of neurons and presynaptic markers and that their order of expression could be preferable when assessing synapse formation using postsynaptic markers. Drebrin A appears earlier than PSD-95 as a marker of postsynaptic structure maturation during human neuronal differentiation [18, 33]. There is still a time lag between mRNA and protein expression and localization. Additional techniques could be necessary to promote the localization of postsynaptic marker proteins in vitro and to induce early postsynaptic structure maturation.

In vitro-expanded hiPSC-NPCs predominantly differentiate into VGLUT2-positive glutamatergic neurons

A substantial amount of excitatory synaptic transmission is mediated by glutamate. VGLUTs carry glutamate to synaptic vesicles, and three isoforms have been identified in humans: VGLUT1, VGLUT2, and VGLUT3 [3436]. Although methods of inducing VGLUT1-positive cells by proliferation at the neural progenitor cell stage have been reported previously [8], there are few similar reports for VGLUT2-positive cells. In this context, the VGLUT2-positive cells derived from hiPSC-NPCs could be a unique finding of our study. VGLUT2-expressing cells have been reported to be located predominantly in the thalamus, hypothalamus (especially the ventromedial nucleus), brainstem, and cortical layer 4 [36], and VGLUT2-positive cells were previously found to be involved in neuropathic pain and central apnea [37, 38]. In the present study, VGLUT1 was not detected by qRTPCR, and few cells were positive for VGLUT1, as determined by immunocytochemistry staining. These findings could be related to the observation that our hiPSC-NPCs highly expressed a ventral hindbrain marker, which is often associated with VGLUT2-positive cells.

Our hiPSC-NPCs showed significant ventral hindbrain markers, which could have been induced ventrally by the inhibition of bone morphogenetic protein (BMP) signaling during the differentiation induction phase of the dual SMAD inhibition protocol and differentiation into the hindbrain system via the EGF and FGF used in the NPC culture. BMP signaling is known to promote dorsalization during the process of differentiation into neurons, and NPCs are thought to be transformed into hindbrain progenitor cells when EGF and FGF are added [39-41]. However, there have been reports of differentiation into the same ventral side of the brain that differentiates into 



SOX1

PAX6

FABP7/BLBP

NES

FOXG1

OTX2

EN1

GBX2

HOXB4

I EMX2

$D L X 2$

NKX2-1

EOMES/TBR2

RELN/reelin

TBR1

I TUBA1A

SLC17A7IVGLUT1

SLC17A6/VGLUT2

GAD1/GAD67

SLC32A1/VGAT

ISL1

CHAT

SLC18A3/VACHT

I $T H$

I TPH2

I SLC18A2/VMAT2

SYP/synaptophysin

SYN1/synapsin I

DLG4IPSD-95

GPHN/gephyrin

$D B N 1 /$ drebrin $A+E$

DBN1/drebrin A

GRIA1/GLUR1

GRIA2/GLUR2

GRIA3

GRIA4

GRIN1/NR1

GRIN2AINR2A

GRIN2BINR2B

GRIN2C

GRIN2D

GFAP

SLC1A3/EAAT1/GLAST

SLC1A2IEAAT2

$A Q P 4$
Neural Progenitor cells

Radial glia

Forebrain

Midbrain

Hindbrain

Spinal cord

Dorsal

Ventral

Intermediate progenitor

Telencephalon

Neuronal

Glutamatergic neuron

GABAergic neuron

Cholinergic neuron

Dopaminergic neuron

Serotonergic neuron

Monoaminergic neuron

Pre-synaptic

Post-synaptic

AMPA receptor

NMDA receptor



Fig. 8 Gene expression analysis of the neural differentiation method at multiple centers. Hierarchical clustering of $1210 B 2$ hiPSC-NPCs and hiPSC-neurons cultured in the absence of NGF (day 49) at multiple centers. A, C, D, and E indicate each center 
GAD67-positive cells [9]. Further investigation is needed to determine which factors lead to differentiation into VGLUT-positive cells. In any case, our method reproducibly achieves differentiation into a cell population centered on VGLUT2-positive cells, which could be useful for pathologies that involve VGLUT2-positive cells, such as neuropathic pain and central apnea [37, 38].

\section{NGF had no significant effect on the differentiation or synapse maturation of hiPSC-NSCs expanded in vitro}

NGF is produced in various parts of the central nervous system [19], and the action of NGF on its receptor, tropomyosin receptor kinase $\mathrm{A}$, is particularly important for the survival of cholinergic nerves in the basal forebrain and has been reported to be involved in dendrite formation, at least in cholinergic neurons $[20,21]$. Thus, we expected the presence or absence of NGF to alter synaptogenesis and differentiation maturation into cholinergic nerves. However, there was no significant effect on the neuronal subtype or gene expression. These findings suggest that the addition of exogenous NGF does not increase the rate of differentiation into cholinergic neurons, at least not in the early stages of neuronal differentiation, and that NGF might not be associated with the formation of excitatory synapses during their formation.

\section{The method for differentiating hiPSC-NPCs expanded in vitro into human neurons is somewhat robust and reproducible}

The reproducibility of this method was confirmed by a multicenter analysis, and the expression of presynaptic molecules and neural differentiation markers was confirmed with high reproducibility. There was also a trend toward the mRNA expression of postsynaptic markers such as drebrin and GLUR. This trend suggests that our culture system is a robust method for inducing neural differentiation and excitatory synapse formation regardless of the experimenter. However, several factors can be improved. The method described herein generally requires a long culturing period for the establishment of the most mature postsynaptic structures. To generate mature synaptic structures earlier from human stem cell-derived neurons in vitro, coculture with mature astrocytes or exogenous factors other than NGF in addition to BDNF may be useful. These points should be addressed in future studies.

\footnotetext{
Abbreviations

hiPSC: Human-induced pluripotent stem cell; NPC: Neural progenitor cell; NSPC: Neural stem/progenitor cell; NGF: Nerve growth factor; VGLUT: Vesicular glutamate transporter; qRT-PCR: Quantitative reverse transcription-polymerase chain reaction; ANOVA: Analysis of variance; ChAT: Choline acetyltransferase; EGF: Epidermal growth factor; FGF: Fibroblast growth factor; GAD67: Glutamate decarboxylase 67; GFAP: Glial fibrillary acidic protein; LIF: Leukemia inhibitory factor; MAP2: Microtubule-associated protein 2; PBS: Phosphate-buffered
}

saline; PSD-95: Postsynaptic density protein 95; TH: Tyrosine hydroxylase; TPH2: Tryptophan hydroxylase 2; TrkA: Tropomyosin receptor kinase A; VAChT: Vesicular acetylcholine transporter.

\section{Supplementary Information}

The online version contains supplementary material available at https://doi. org/10.1186/s13041-021-00851-1.

Additional file 1: Table 1. Primers for SYBR Green-based qRT-PCR

Additional file 2: Table 2. Antibodies used in this study.

Additional file 3: Table 3. Antibodies used in Western bold analysis.

Additional file 4: Figure S1. Gene expression analysis of pre- and postsynaptic markers during the neuronal differentiation of hiPSC-NPCs in the absence and presence of NGF. Dot and box plots showing the expression of postsynaptic markers (drebrin $A+E$ and drebrin $A$ ) and a presynaptic marker (synaptophysin) during neuronal differentiation. Statistical analysis was performed using ANOVA with the post hoc Tukey-Kramer method and Student's t-tests $\left({ }^{* * *} p<0.001,{ }^{* *} p<0.01,{ }^{*} p<0.05\right)$. Figure S2. Protein expression of postsynaptic markers in terminally differentiated 1210B2 hiPSC neurons. Time-dependent immunofluorescence images of BIII-tubulin, MAP2, total drebrin, drebrin A, synaptophysin, and PSD-95 in $1210 B 2$ cells in the absence of NGF. The nuclei were stained with DAPI (blue). Scale bar: 20 um. Figure S3. Positive control of immunostaining image. SKY neurons cultured for 21 days were used as a positive control for the PSD-95 antibody. The staining was confirmed by setting up a control. Scale bar: 20 um. Figure S4. Immunostaining images of preand postsynaptic markers on day 49 in hiPSC neurons in the presence and absence of NGF. Immunofluorescence images of MAP2, drebrin A, synaptophysin, and PSD-95 in 1210B2 and 1201C1 cells in the presence and absence of NGF on day 49. The nuclei were stained with DAPI (blue). Scale bar: $20 \mu \mathrm{m}$. Figure S5. Western blot analysis of presynaptic proteins in hiPSC neurons on day 0, day 49, and rat cerebrum tissue lysate. For the rat cerebrum tissue sample, $10 \mu \mathrm{g}$ of protein per well was used, and for the other samples, $30 \mu \mathrm{g}$ of protein per well was used for analysis. Figure S6. Relative protein expression was assessed by Western blot analysis. The protein expression levels of pre- and postsynaptic marker were determined by the $\beta$-Actin expression level used as an internal control and then shown as the relative ratio to the expression level in the control rat cerebrum lysate. UD undetermined.

\section{Acknowledgements}

The authors thank Ms. Ai Takada, Ms. Miho Sumida, Ms. Ema Yoshioka, Ms. Yukako Handa, Ms. Asako Katsuma, Ms. Atsuyo Yamamoto, Mr. Daisuke Kanematsu and Ms. Eriko Urano for their technical support.

\section{Authors' contributions}

KT conceived and designed the experiments, performed the experiments, analyzed the data, contributed reagents/materials/analysis tools, wrote the paper, prepared the figures and tables, and reviewed drafts of the paper. HF conceived and designed the experiments, contributed reagents/materials/ analysis tools, and reviewed drafts of the paper. TS conceived and designed the experiments, supervised the research, contributed reagents/materials/ analysis tools, and reviewed drafts of the paper. HO, HY, MH, NK, NT, TN, YS, HH performed an experiment, contributed reagents/materials/analysis tools, and reviewed drafts of the paper. KB, HM supervised the research and reviewed drafts of the paper. YS conceived and designed the experiments, supervised the research, performed the experiments, analyzed the data, and reviewed drafts of the paper. TS conceived and designed the experiments, performed an experiment, contributed reagents/materials/analysis tools, and reviewed drafts of the paper. YK conceived and designed the experiments, supervised the research, and reviewed drafts of the paper. All authors read and approved the final manuscript.

\section{Funding}

This study was supported by the Japan Agency for Medical Research and Development (AMED) (Y.K., JP21bm0204001 and JP21bm0804024) and 
(T.S., JP19bk0104077). This study was also supported by JSPS KAKENHI (Y.K., JP18K08958).

\section{Availability of data and materials}

All data generated and/or analyzed in this study are included in this published article

\section{Declarations}

\section{Ethics approval and consent to participate}

This study was conducted in accordance with the principles of the Declaration of Helsinki. The use of hiPSCs was approved by the Osaka National Hospital hN-NSPCs and hiPSCs ethics committee (Nos. 110, 120, and 146).

\section{Consent for publication}

Not applicable.

\section{Competing interests}

The authors have no competing interests to declare.

\section{Author details}

'Department of Neurology, Graduate School of Medicine, Osaka University, Suita, Osaka 565-0871, Japan. ${ }^{2}$ Division of Stem Cell Research, Department of Biomedical Research and Innovation, Institute for Clinical Research, National Hospital Organization Osaka National Hospital, Osaka, Osaka 540-0006, Japan. ${ }^{3}$ Department of Laboratory Sciences, Gunma University Graduate School of Health Sciences, Maebashi, Gunma 371-8514, Japan. ${ }^{4}$ Department of Neurobiology and Behavior, Gunma University Graduate School of Medicine, Maebashi, Gunma 371-8511, Japan. ${ }^{5}$ Faculty of Social Welfare, Gunma University of Health and Welfare, Maebashi, Gunma 371-0823, Japan. ${ }^{6}$ School of Medicine, International University of Health and Welfare, Narita, Chiba 286-8686, Japan. ${ }^{7}$ Department of Food Science and Nutrition, Faculty of Food and Health Sciences, Showa Women's University, Setagaya-ku, Tokyo 154-8533, Japan. ${ }^{8}$ Laboratory of Biopharmaceutical and Regenerative Sciences, Graduate School of Medical Life Science, Yokohama City University, Yokohama, Kanagawa 230-0045, Japan. ${ }^{9}$ Department of Brain Tumor Biology, Brain Research Institute, Niigata University, Niigata, Niigata 951-8585, Japan. ${ }^{10}$ Laboratory of Molecular Neuropharmacology, Graduate School of Pharmaceutical Sciences, Osaka University, Suita, Osaka 565-0871, Japan. ${ }^{11}$ Department of Bioscience, Faculty of Life Sciences, Tokyo University of Agriculture, Setagaya-ku, Tokyo 156-8502, Japan. ${ }^{12}$ Graduate School of Integrated Sciences for Life, Hiroshima University, Higashi-Hiroshima, Hiroshima 739-8521, Japan. ${ }^{13}$ Molecular Research Center for Children's Mental Development, United Graduate School of Child Development, Osaka University, Suita, Osaka 565-0871, Japan. ${ }^{14}$ Division of Bioscience, Institute for Datability Science, Osaka University, Suita, Osaka 565-0871, Japan. ${ }^{15}$ Open and Transdisciplinary Research Initiatives, Osaka University, Suita, Osaka 565-0871, Japan. ${ }^{16}$ Department of Molecular Pharmaceutical Sciences, Graduate School of Medicine, Osaka University, Suita, Osaka 565-0871, Japan. ${ }^{17}$ Endowed Laboratory of Human Cell-Based Drug Discovery, Graduate School of Pharmaceutical Sciences, The University of Tokyo, Bunkyo-ku, Tokyo 113-0033, Japan. ${ }^{18}$ Division of Regenerative Medicine, Department of Biomedical Research and Innovation, Institute for Clinical Research, National Hospital Organization Osaka National Hospital, 2-1-14 Hoenzaka, Chuo-ku, Osaka, Osaka 540-0006, Japan. ${ }^{19}$ Department of Neurosurgery, National Hospital Organization Osaka National Hospital, Osaka, Osaka 540-0006, Japan.

Received: 11 February 2021 Accepted: 4 September 2021 Published online: 11 October 2021

\section{References}

1. Logan S, Arzua T, Canfield SG, Seminary ER, Sison SL, Ebert AD, et al. Studying human neurological disorders using induced pluripotent stem cells: from 2D monolayer to 3D organoid and blood brain barrier models. Compr Physiol. 2019;9(2):565-611.

2. Cavalli E, Battaglia G, Basile MS, Bruno V, Petralia MC, Lombardo SD, et al. Exploratory analysis of iPSCS-derived neuronal cells as predictors of diagnosis and treatment of Alzheimer disease. Brain Sci. 2020;10(3):166.
3. Fukusumi H, Handa Y, Shofuda T, Kanemura Y. Evaluation of the susceptibility of neurons and neural stem/progenitor cells derived from human induced pluripotent stem cells to anticancer drugs. J Pharmacol Sci. 2019;140(4):331-6.

4. Hoffman GE, Hartley BJ, Flaherty E, Ladran I, Gochman P, Ruderfer DM, et al. Transcriptional signatures of schizophrenia in hiPSC-derived NPCs and neurons are concordant with post-mortem adult brains. Nat Commun. 2017;8(1):2225.

5. Tukker AM, Wijnolts FMJ, de Groot A, Westerink RHS. Human iPSC-derived neuronal models for in vitro neurotoxicity assessment. Neurotoxicology. 2018;67:215-25.

6. Fukusumi H, Handa Y, Shofuda T, Kanemura Y. Small-scale screening of anticancer drugs acting specifically on neural stem/progenitor cells derived from human-induced pluripotent stem cells using a time-course cytotoxicity test. PeerJ. 2018;6:e4187.

7. Xylaki M, Atzler B, Outeiro TF. Epigenetics of the synapse in neurodegeneration. Curr Neurol Neurosci Rep. 2019;19(10):72.

8. D'Aiuto L, Zhi Y, Kumar Das D, Wilcox MR, Johnson JW, McClain L, et al. Large-scale generation of human iPSC-derived neural stem cells/early neural progenitor cells and their neuronal differentiation. Organogenesis. 2014;10(4):365-77.

9. Falk A, Koch P, Kesavan J, Takashima Y, Ladewig J, Alexander M, et al. Capture of neuroepithelial-like stem cells from pluripotent stem cells provides a versatile system for in vitro production of human neurons. PLoS ONE. 2012;7(1):e29597.

10. Nadadhur AG, Emperador Melero J, Meijer M, Schut D, Jacobs G, Li KW, et al. Multi-level characterization of balanced inhibitory-excitatory cortical neuron network derived from human pluripotent stem cells. PLoS ONE. 2017;12(6):e0178533.

11. Wang H, Hu L, Liu C, Su Z, Wang L, Pan G, et al. 5-HT2 receptors mediate functional modulation of GABAa receptors and inhibitory synaptic transmissions in human iPS-derived neurons. Sci Rep. 2016;6:20033.

12. Harris KM, Jensen FE, Tsao B. Three-dimensional structure of dendritic spines and synapses in rat hippocampus (CA1) at postnatal day 15 and adult ages: implications for the maturation of synaptic physiology and long-term potentiation. J Neurosci. 1992;12(7):2685-705.

13. Wilson ES, Newell-Litwa K. Stem cell models of human synapse development and degeneration. Mol Biol Cell. 2018;29(24):2913-21.

14. Ho BX, Pek NMQ, Soh BS. Disease modeling using 3D organoids derived from human induced pluripotent stem cells. Int J Mol Sci. 2018;19(4):936.

15. Kojima N. Molecular cloning of drebrin: progress and perspectives. Adv Exp Med Biol. 2017;1006:25-36.

16. Toda M, Shirao T, Minoshima S, Shimizu N, Toya S, Uyemura K. Molecular cloning of cDNA encoding human drebrin E and chromosomal mapping of its gene. Biochem Biophys Res Commun. 1993;196(1):468-72.

17. Kojima N, Shirao T, Obata K. Molecular cloning of a developmentally regulated brain protein, chicken drebrin $A$ and its expression by alternative splicing of the drebrin gene. Brain Res Mol Brain Res. 1993;19(1-2):101-14.

18. Takahashi H, Sekino Y, Tanaka S, Mizui T, Kishi S, Shirao T. Drebrin-dependent actin clustering in dendritic filopodia governs synaptic targeting of postsynaptic density-95 and dendritic spine morphogenesis. J Neurosci. 2003:23(16):6586-95.

19. Rocco ML, Soligo M, Manni L, Aloe L. Nerve growth factor: early studies and recent clinical trials. Curr Neuropharmacol. 2018;16(10):1455-65.

20. Markova EG, Isaev NK. Effects of nerve growth factor on the development of the dendritic system of cholinergic neurons in dissociated culture of the rat septum. Biull Eksp Biol Med. 1992;113(3):318-20.

21. Cuello AC, Pentz R, Hall H. The brain NGF metabolic pathway in health and in Alzheimer's pathology. Front Neurosci. 2019;13:62.

22. Sugai K, Fukuzawa R, Shofuda T, Fukusumi H, Kawabata S, Nishiyama Y, et al. Pathological classification of human iPSC-derived neural stem/ progenitor cells towards safety assessment of transplantation therapy for CNS diseases. Mol Brain. 2016:9(1):85.

23. Fukusumi H, Shofuda T, Bamba Y, Yamamoto A, Kanematsu D, Handa $Y$, et al. Establishment of human neural progenitor cells from human induced pluripotent stem cells with diverse tissue origins. Stem Cells Int. 2016;2016:7235757.

24. Nakagawa M, Taniguchi Y, Senda S, Takizawa N, Ichisaka T, Asano K, et al. A novel efficient feeder-free culture system for the derivation of human induced pluripotent stem cells. Sci Rep. 2014;4:3594. 
25. Okita K, Yamakawa T, Matsumura Y, Sato Y, Amano N, Watanabe A, et al. An efficient nonviral method to generate integration-free humaninduced pluripotent stem cells from cord blood and peripheral blood cells. Stem Cells. 2013;31(3):458-66.

26. Kanemura Y, Mori H, Kobayashi S, Islam O, Kodama E, Yamamoto A, et al. Evaluation of in vitro proliferative activity of human fetal neural stem/ progenitor cells using indirect measurements of viable cells based on cellular metabolic activity. J Neurosci Res. 2002;69(6):869-79.

27. Shofuda T, Fukusumi H, Kanematsu D, Yamamoto A, Yamasaki M, Arita N, et al. A method for efficiently generating neurospheres from humaninduced pluripotent stem cells using microsphere arrays. NeuroReport. 2013;24(2):84-90.

28. Kobayashi Y, Okada T, Miki D, Sekino Y, Koganezawa N, Shirao T, et al. Properties of primary cilia in melanin-concentrating hormone receptor 1-bearing hippocampal neurons in vivo and in vitro. Neurochem Int. 2021;142:104902.

29. Mitsuoka T, Hanamura K, Koganezawa N, Kikura-Hanajiri R, Sekino Y, Shirao T. Assessment of NMDA receptor inhibition of phencyclidine analogues using a high-throughput drebrin immunocytochemical assay. J Pharmacol Toxicol Methods. 2019;99:106583.

30. Isoda M, Kohyama J, Iwanami A, Sanosaka T, Sugai K, Yamaguchi R, et al. Robust production of human neural cells by establishing neuroepitheliallike stem cells from peripheral blood mononuclear cell-derived feederfree iPSCs under xeno-free conditions. Neurosci Res. 2016;110:18-28.

31. Cotterill E, Charlesworth P, Thomas CW, Paulsen O, Eglen SJ. A comparison of computational methods for detecting bursts in neuronal spike trains and their application to human stem cell-derived neuronal networks. J Neurophysiol. 2016;116(2):306-21.

32. Odawara A, Katoh H, Matsuda N, Suzuki I. Physiological maturation and drug responses of human induced pluripotent stem cell-derived cortical neuronal networks in long-term culture. Sci Rep. 2016;6:26181.

33. Mizui T, Takahashi H, Sekino Y, Shirao T. Overexpression of drebrin A in immature neurons induces the accumulation of F-actin and PSD-95 into dendritic filopodia, and the formation of large abnormal protrusions. Mol Cell Neurosci. 2005;30(1):149-57.

34. Bellocchio EE, Reimer RJ, Fremeau RT Jr, Edwards RH. Uptake of glutamate into synaptic vesicles by an inorganic phosphate transporter. Science. 2000;289(5481):957-60.

35. Aihara $Y$, Mashima H, Onda H, Hisano S, Kasuya H, Hori T, et al. Molecular cloning of a novel brain-type $\mathrm{Na}(+)$-dependent inorganic phosphate cotransporter. J Neurochem. 2000;74(6):2622-5.

36. Fremeau RT Jr, Voglmaier S, Seal RP, Edwards RH. VGLUTs define subsets of excitatory neurons and suggest novel roles for glutamate. Trends Neurosci. 2004;27(2):98-103.

37. Wallén-Mackenzie A, Gezelius H, Thoby-Brisson M, Nygård A, Enjin A, Fujiyama F, et al. Vesicular glutamate transporter 2 is required for central respiratory rhythm generation but not for locomotor central pattern generation. J Neurosci. 2006;26(47):12294-307.

38. Moechars D, Weston MC, Leo S, Callaerts-Vegh Z, Goris I, Daneels G, et al. Vesicular glutamate transporter VGLUT2 expression levels control quantal size and neuropathic pain. J Neurosci. 2006;26(46):12055-66.

39. Bilican B, Livesey MR, Haghi G, Qiu J, Burr K, Siller R, et al. Physiological normoxia and absence of EGF is required for the long-term propagation of anterior neural precursors from human pluripotent cells. PLOS ONE. 2014;9(1):e85932.

40. Zhou S, Ochalek A, Szczesna K, Avci HX, Kobolák J, Varga E, et al. The positional identity of iPSC-derived neural progenitor cells along the anterior-posterior axis is controlled in a dosage-dependent manner by bFGF and EGF. Differentiation. 2016;92(4):183-94.

41. Stern CD. Initial patterning of the central nervous system: how many organizers? Nat Rev Neurosci. 2001;2(2):92-8.

\section{Publisher's Note}

Springer Nature remains neutral with regard to jurisdictional claims in published maps and institutional affiliations.
Ready to submit your research? Choose BMC and benefit from:

- fast, convenient online submission

- thorough peer review by experienced researchers in your field

- rapid publication on acceptance

- support for research data, including large and complex data types

- gold Open Access which fosters wider collaboration and increased citations

- maximum visibility for your research: over $100 \mathrm{M}$ website views per year

At BMC, research is always in progress.

Learn more biomedcentral.com/submissions 